Research Paper

\title{
Neurophysiologic Measurement of Continuity in the Sleep of Fetuses during the Last Week of Pregnancy and in Newborns
}

\author{
Adrián Poblano ${ }^{1,2}$, Reyes Haro ${ }^{1}$, and Carmina Arteaga ${ }^{2}$ \\ 1. Clinic of Sleep Disorders, School of Medicine, National University of Mexico, Mexico City, Mexico \\ 2. Laboratory of Cognitive Neurophysiology, National Institute of Rehabilitation. Mexico City, Mexico \\ Correspondence to: A. Poblano, M.D., M.Sc., D.Sc. Dr. Balmis 148, Col. Doctores, Deleg. Cuauhtémoc, 06720, Mexico City, Mexico. \\ Phone/Fax: (+52) (55) 5489-6599; E-mail: drdeaf@starmedia.com
}

Received: 2007.08.01; Accepted: 2007.12.12; Published: 2007.12.13

Our aim was to measure the correlation between fetal electrocardiographic (FECG) recordings of low-risk pregnancies and polysomnographic (PSG) study parameters in low-risk infants born at term as a measurement of perinatal sleep-development continuity.

We designed a short, prospective, observational follow-up of physiologic parameters between fetuses and newborns. We studied 10 fetuses from low-risk pregnant female out-patients and the same subjects as low-risk newborns delivered at term. Fetal state (FS) was defined in FECG recordings reassembling the following: fetal state I (quiet sleep or QS); fetal state II (active sleep or AS); fetal state III (quiet waking), and fetal state IV (active waking). Percentages of AS, QS, and wakefulness in PSG studies of newborns were also determined.

Comparisons of FS I with QS showed a significant reduction in QS, while comparison of FS II with AS showed significant reduction in AS. Negative correlations were found between FS I with QS, and FS II with AS. Number of cycles in FECG recordings and PSG sleep cycles also demonstrated significant correlation.

In conclusion our data showed partial but significant sleep function continuity from fetal to neonatal period.

Key words: Sleep development, Fetuses, Newborns, Fetal electrocardiographic recordings, Polysomnography.

\section{INTRODUCTION}

Continuity of central nervous system (CNS) functions in fetuses from pregnancy to early post-natal life has received scarce attention [1]. One example is the development of the awake-sleep cycle, which originates during the fetal and extends to the neonatal period. Early sleep development possesses an ontogenetic role and is necessary for cognitive development and other adaptation functions, such as memory and attention [2-4].

With the development and clinical availability of external fetal heart-rate monitoring equipment, renewed interests arose in the application of this technique in an attempt to better define the prenatal fetal sleep function. Due to the fact that it is an easy and non-invasive procedure and that different working groups have contributed to a growing field of knowledge, fetal electrocardiographic recording (FECG) becomes one of the better tools to define fetal behavior. According to fetal heart-rate variability recordings and ultrasonographic (US) observations, four fetal behavioral states have been identified in humans at 36 weeks of gestation [5]: State 1 was defined by absence of eye movements, stable heart rate pattern with narrow oscillation bandwidth, and infrequent gross body movements; state 2 was defined by presence of continuous eye movements and wider heart rate oscillation bandwidth with frequent accelerations and gross movements; state 3 was defined by presence of eye movements, a wider oscillation bandwidth than observed in state 1 but more regular than that observed in state 2 , and state 4 defined by presence of eye movements, gross body movements, and unstable heart rate pattern with large and prolonged accelerations, often fused into sustained tachycardia [6]. Fetal states are in agreement with those observed in polysomnographic (PSG) recording-associated in newborns [7-10], these approximating reassembly quiet sleep (fetal state I), active sleep (fetal state II), quiet waking (fetal state III), and active waking (fetal state IV), respectively.

On the other hand, sleep recording by PSG is the most adequate electrophysiologic test to identify sleep patterns in pre-term and at-term newborns. Three sleep states can be observed in premature infants: active sleep (AS) with presence of rapid eye movements; quiet sleep (QS) without rapid eye movements, and indeterminate sleep (IS). In low-risk at-term infants, we found mainly AS, and QS [10]. In AS, the electroencephalogram (EEG) is low amplitude, 
rapid eye movements can be clearly recorded, phasic electromyographic activity occurs irregularly, and irregular and fast heart rate is accompanied by irregular respiration. In QS, the EEG contains periodic burst of waves with high amplitude (i.e. tracé alternant pattern), eyes remain without rapid movements, no body movements occur, heart rate is stable and slow, and respiration is regular. IS possesses mixed characteristics of both, AS and QS. To our knowledge, no studies have sought concordance measurements among fetal states and later with post-natal AS and QS states.

Our objective was to carry out observations in a short prospective follow-up of fetuses studied from late pregnancy to early post-natal life, measuring correlations between FECG parameters of low-risk pregnancies and their fetuses and subsequently calculating correlation with PSG studies in the same low-risk at-term infants as a measurement of perinatal sleep-development continuity, attempting to determine whether there are significant changes in newborn sleep when compared with fetal sleep.

\section{MATERIALS AND METHODS}

\section{Subjects}

Healthy women between 19 and 31 years of age in the first trimester of pregnancy who required specialized assistance in a tertiary-level health institute for high-risk pregnancies in Mexico City were recruited; the sample was selected non-randomized between women requesting a consultation and having a non-complicated pregnancy. Age of pregnancy was determined by last menstruation date and was verified in infants at birth by Capurro method [11]. Ten pregnant women were selected into the study; they were predominantly of lower socioeconomic status, residing in the Valley of Mexico, with a median of one prior birth and a mean age of $24.2 \pm 3.6$ years (average \pm standard deviation [SD]). Women were carefully selected, only those who had no risk factors during pregnancy such as: being a too-young or a too-old mother ( $<19$ and $>31$ years of age), diabetic, with high blood pressure (diastolic pressure $>90 \mathrm{~mm} \mathrm{Hg}$ ), infectious diseases such as toxoplasmosis and others during pregnancy, vaginal bleeding, and women taking regular medication or declared as abusive of alcohol, tobacco or other drugs during pregnancy were allowed to participate in the study.

We studied the 10 newborns of these 10 women selected. Infants were delivered at term (three were males), without any complications. Inclusion criteria in infants were the following: born with a 5-min Apgar score of 9 or higher; birth weight between 2,500 and $4,000 \mathrm{~g}$; gestation age ranging from 38-41 weeks, and without major congenital anomalies; these criteria ensured studying infants at low risk for neurologic damage. Clinical characteristics of the group are shown in Table 1. The Institutional Review Board approved the protocol, and adult patients signed informed consent for their participation and that of their infants.

TABLE 1. Clinical characteristics of infants

\begin{tabular}{|cc}
\hline Variable & $\mathrm{X} \pm \mathrm{SD}$ \\
Age at birth (weeks) & $41.0 \pm 1.1$ \\
\hline Weight $(\mathrm{g})$ & $3543 \pm 439$ \\
\hline Height $(\mathrm{cm})$ & $49.4 \pm 1.8$ \\
\hline Apgar 1 & 8 (median) \\
\hline Apgar 5 & 9 (median) \\
\hline
\end{tabular}

$\mathrm{SD}$, standard deviation.

\section{Fetal Electrocardiographic Recordings}

FECR monitoring began following admission and preparation of the mother at the department of fetal medicine of the hospital. Average and standard deviation of fetus age at time of study was $39.4 \pm 1.4$ weeks of gestation. Studies were carried out during $8 \mathrm{~h}$ for research purposes in a silent and dimly lit room adjacent to that containing study equipment between 7 A.M. and 3 P.M. The mother's behavior was observed continuously by video-camera. Maternal electrocardiogram was recorded with two disposable electrodes placed beneath the clavicles. The technique for long-term FECR was performed after the Eisenberg de Smoler and Karchmer study modified for recording with additional electrodes [12]. Eight silver-plate disk electrodes were placed in a circle on abdominal skin over uterus; these were symmetrically distributed with a $45^{\circ}$ angle between neighboring electrodes; signals were amplified, transmitted to polygraph Grass $78 \mathrm{D}$ equipment, and recorded on a 3968-D Hewlett-Packard FM tape recorder for off-line analysis. Fetal states were defined after Nijhuis et al [13].

\section{Polysomnography}

PSG studies were performed at the laboratory of neurophysiology in the clinic. Average and standard deviation of newborns age at time of study was $6.0 \pm$ 2.0 days of life. PSG studies had an 8-h duration to match the time of PSG recordings between 7 A.M. and 3 P.M. Electrode impedance was always $<5 \mathrm{~K}$-ohms. Recordings were performed on a digital polysomnographer (Nicolet, Madison, WI, USA) with eight channels for electroencephalography (EEG), an additional channel for electromyography (EMG), two additional channels for electro-oculography (EOG), one additional channel for electrocardiography (ECG), and four channels for respiration (pneumography). 
Gold disk electrodes were placed according to international system modified for newborns [14]. We used bipolar EEG montage as follows: Fp1-C3; C3- O1; Fp1-T3; T3-O1; Fp2-C4; C4-O2; Fp2-T4, and T4-O2. Band pass filters were set between 0.1 and 35 Hertz (Hz).

EMG recording was performed at the chin and was qualified as presence or absence of burst of myogenic potentials. EOG activity was measured at external angles of eye and qualified as presence or absence of eye movements. ECG was recorded by placing electrodes on thorax under clavicles and was evaluated for variations in QRS frequency to determine bradycardia. Respiration was measured by means of a nasal thermistor and with respiratory thoracic and abdominal bands [15].

The technician described any change in infant behavior during the recording session according to behavioral-state criteria [16]. When the infant was awake for periods $>1$ min or was feeding, recording was no stopped, but this time was counted as awake time. PSG studies were evaluated using 30-sec epochs to identify active (AS) or quiet sleep (QS) $[14,17]$.

\section{Data Analyses}

Descriptive analysis was performed to determine average and standard deviation for continuous variables, and percentages were calculated for binomial variables. Paired Student $t$ tests were conducted to search for differences among numerical variables. We calculated Pearson's correlations between the results of FECR and PSG studies. Level of statistical significance was $\leq 0.05$ [18].

\section{RESULTS}

During fetal heart-rate recordings by FECR, the fetus spent an average of $25.7 \pm 7.5$ (\% of time) in fetal state I, while the fetus spent $71.8 \pm 10.0$ (\% of time) in fetal state II; overall, fetal states I plus II (FS I + FS II) spent were $97.6 \pm 3.8$ (\% of time) of recordings. Number of studied cycles comprised $4.7 \pm 2.8$ cycles. Quantitative analyses of duration of fetuses in each fetal state are presented in Table 2.

In PSG recordings, infants spent $20.7 \pm 6.7$ (\% of time) in QS, while they spent $55.9 \pm 13.2$ (\% of time) in AS. Total sleeping time was $76.7 \pm 15.5$ (\% of time). Number of cycles studied was $4.5 \pm 0.8$ (see Table 3 ).

Comparison of percentage spent by fetuses in FS I with QS spent by newborns showed a significant reduction of QS $(t=4.26, p<0.001)$. Comparison between average time spent between fetal state II with AS showed a significant reduction of AS $(t=28.90, p$ $<0.001)$.

Correlations between FECG recordings and PSG studies in quantitative and percentage measurements in fetuses and infants showed significant results between fetal state I and QS $(r=-0.79, p=0.01)$, fetal state II and AS $(r=-0.88, p=0.001)$. The scatterplot of a typical correlation is shown in Figure 1. Number of cycles in FECG recordings and in PSG studies also showed significant correlation $(r=-0.78, p=0.01)$. Quadratic $r$ values are shown in Table 4.

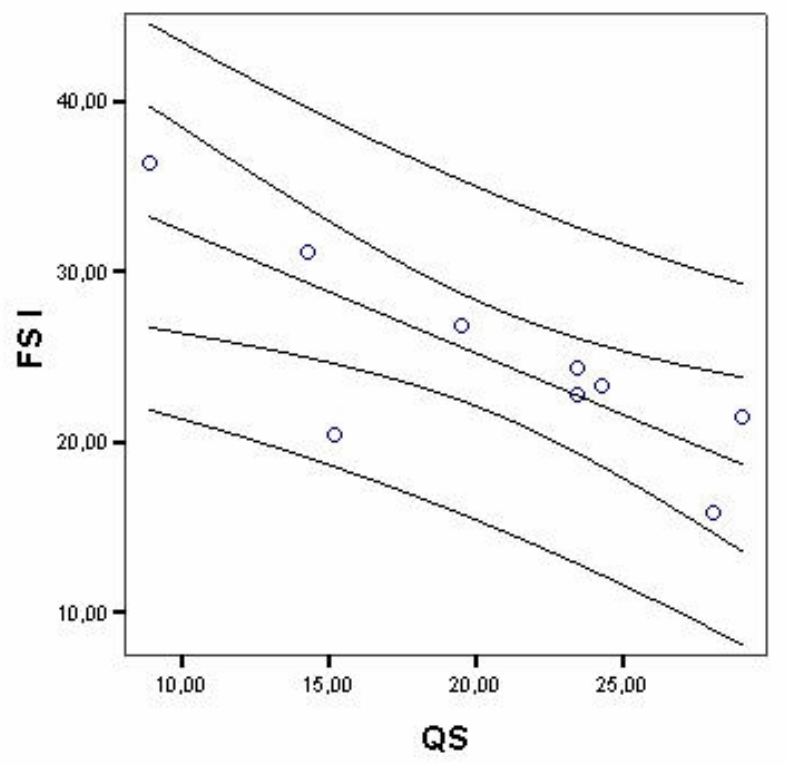

Figure 1. Typical scatterplot example showing correlation between percentages of Fetal state I duration (FS I) and Quiet sleep (QS) in newborns, with regression line and confidence intervals (95\%) with significant association among variables.

TABLE 2. Duration spent in each fetal sleep state by fetuses

\begin{tabular}{|c|c|c|}
\hline Variable & $\mathrm{X} \pm \mathrm{SD}$ & $\%$ \\
\hline State I (min) & $123.6 \pm 31.6$ & 25.7 \\
\hline State II (min) & $345.2 \pm 29.1$ & 71.8 \\
\hline $\begin{array}{c}\text { State I + State II } \\
\text { (min) }\end{array}$ & $468.8 \pm 18.2$ & 97.6 \\
\hline
\end{tabular}

$\mathrm{SD}$, standard deviation. \% percentage

TABLE 3. Quiet and active sleep duration in PSG recordings in infants

\begin{tabular}{|c|c|c|}
\hline Variable & $\mathrm{X} \pm \mathrm{SD}$ & $\%$ \\
\hline QS (min) & $99.6 \pm 32.4$ & 20.7 \\
\hline AS (min) & $268.8 \pm 63.7$ & 55.9 \\
\hline QS + AS (Min) & $368.6 \pm 74.5$ & 76.7 \\
\hline
\end{tabular}

$\mathrm{SD}$, standard deviation. \% percentage

TABLE 4. Correlations between significant FE-CT sleep measurements and infant PSG measurements

\begin{tabular}{|c|c|c|}
\hline $\begin{array}{c}\text { Correlations between fetal and newborn } \\
\text { sleep states }\end{array}$ & $r$ & $r^{2}$ \\
\hline $\begin{array}{c}\text { Fetal state I-Quiet sleep } \\
\text { Fetal state II-Active sleep }\end{array}$ & -0.79 & 0.62 \\
\hline $\begin{array}{c}\text { Number of fetal sleep cycles-Number of } \\
\text { PSG sleep cycles }\end{array}$ & -0.88 & 0.77 \\
\hline
\end{tabular}




\section{DISCUSSION}

Significant correlations between low-risk fetus and healthy newborn sleep states in infants born at term were found in the present work. Correlation analysis was employed as a sleep-function continuity measurement from fetal to neonatal period as index of development in this brief, but important moment. Quadratic value of $r$ calculations were found at between 0.60 and 0.77 (see Table 4), suggesting that between three- and four-fifths of variance can be explained by correlation analyses. Although this is a significant value, it suggests that other variables after birth begin to play significant roles in sleep development, and add to the general knowledge that there are low but significant differences between the sleep of fetuses and newborns, according to our hypotheses quoted previously.

Few studies have examined associations between fetal state coincidence and post-natal sleep function. Junge (1979) studied normal-at-term fetuses and a small group of abnormal fetuses by means of fetal heart-rate measurement performed by external cardiotocogram $\mathrm{ca} 4$ days before birth; heart rate and respiration were recorded again in the same subjects with the exception of two anencephalic infants who died. Infant state behavior was qualified visually and polygraphically. The author concluded that in the newborn infant state-related heart-rate pattern, changes occur identically to those of heart-rate pattern in fetus [19]; nonetheless, no correlation calculation was performed.

Pillai and James (1990) performed comparisons between ultrasound behavioral recordings of 18 healthy term fetuses and recordings of the same variables of the same individuals 3 and 5 days postnatally. Statistical comparison of the frequency of eye, limb, and body movements revealed that fetal states $1 \mathrm{~F}$ and $2 \mathrm{~F}$ were comparable with quiet sleep (S1) and rapid eye movement (REM) sleep (S2), respectively, in the newborn. Comparisons between fetal states and wakefulness were less clear-cut [20].

Groome et al. (1997) studied 30 low-risk fetuses at term by means of fetal heart-rate monitoring and again as neonates at 2 weeks post-natal age by heart-rate recordings and behaviorally [21]; sleep states were qualified according to Thoman and Whitney criteria [22]. The authors observed that the time spent in a sleep period was distributed mainly between QS and AS in virtually identical proportions for fetuses and neonates; notwithstanding this, the only within-subject consistency between fetal and neonatal periods comprised the duration of complete QS epochs. These authors performed no correlation calculation.

di Pietro et al. (2002) followed 52 normally developing fetuses at 24, 30, and 36 weeks of gestation employing an actocardiograph; after birth, behavioral-structured neonatal assessment was carried out in 41 infants [3]. The authors observed that intra-fetal stability emerges at 36 weeks of gestation. Fetuses with higher concordance at 36 weeks included infants displaying better state regulation, including more alertness and orientation, less burden of maintaining attention, less irritability, better regulatory capacity, a greater range of available states, and those who were significantly more likely to maintain control during the most aversive portions of the examination. These results support that fetal-state regulation is preserved across the prenatal and neonatal periods. No correlation analyses were performed by the authors.

Comparison between the previously mentioned studies and our data cannot be effected due to differences in methodology, type of fetal and neonatal sleep measurement, and different age at time of infant study [3, 19-21]. However, all quoted studies observed a close relationship between sleep states in at-term fetuses and sleep in newborns, and are in good agreement with our data.

We utilize correlation analyses for weighting the force-of-association between sleep organization in both fetuses and infants. Correlation coefficient values were strong, meaning significant dependence between variables in negative direction. A greater amount of fetal sleep correlates with a lesser amount of sleep in newborns. Afterward we calculated $r^{2}$ to predict the amount of variance that can be attributed to correlation between sleep architecture in these two periods of life; however, sleep variability from fetuses to newborns explain nearly four-fifths of variance, suggesting that solely one-fifth must be explained by other ways and a partial continuity of sleep development.

Maturational changes in sleep-state organization continue both before and after birth, but under different environmental influences. By approximately 36- 38 weeks, state variables become sufficiently synchronized for well-defined behavioral states to be identified in the majority of normal human fetuses [13, 20]. Similarly, postnatal studies suggest that state differentiation may begin early. Recent studies examining state organization in preterm infants report an increase in the proportion of time spent in QS and a decrease in the proportion of time spent in AS as term age is approached [23]. The 38-40-weeks-of-age human fetus spends only $\mathrm{ca} 5 \%$ of its time awake (fetal states III and IV); the proportion of time spent in these states may increase to approximately $20 \%$ in fetus undelivered at 41 weeks of gestation. Newborn term 
infants exhibit both ultradian and diurnal cyclicity in their sleep patterns on postnatal days 1 and 2, and significant maturational changes in the proportion of QS and AS continue to occur between weeks 40 and 45 postconceptional age.

Time of first clear appearance of behavioral states in-utero has been reported as between 36 and 38 weeks of gestation $[3,13]$. In premature newborns, behavioral states are well-defined only after 36 weeks of gestation [23], the time at which this study was performed. At this time, brain morphologic developmental change is rapid [24]. Development of neuronal cortical networks in premature and early infancy has been revised; the authors found that synaptic density increases faster in auditory cortex, where the maximum is reached rapidly at the post-natal age of 3 months [25]; synaptogenesis occurs concurrently with dendritic and axonal growth and with myelination of the sub-cortical white matter [26]. Brain 2-deoxyglucose utilization measured by positron emission tomography (PET) in infants revealed that glucose uptake was highest in sensorimotor cortex, thalamus, midbrain-brainstem, and cerebellar vermis [27, 28]; some of these structures are involved in sleep regulation. All these rapid maturational morphologic and functional changes in parallel with sleep-development trends may aid to explaining the differences between both fetus sleep parameters and those of the neonate.

Based on our findings, we conclude that there are differences in sleep-related brain structure development between prenatal and postnatal periods. Our data support the hypothesis of the ontogenetic role of sleep development in perinatal period (especially AS) [29]; we observed that the more the fetus sleeps, the less newborn sleeps. This finding can be explained that fetuses with higher percentages of sleep had spent more time in developing anatomic and chemical brain networks for postnatal sleep control as a newborn than those who slept less time, exhibiting a more mature sleep pattern. The latter hypothesis deserves more attention in future works. From a developmental perspective, AS has generally been regarded as the most ontogenetic of the sleep states; animals with a structurally immature brain at birth are in AS longer than animals with a relatively mature brain [30].

Nevertheless, the main limitations of the study include the small number of patients studied and the short duration of follow-up; thus, our results must be considered tendencies, and not strong conclusions. Future studies regarding correlation between fetal and neonatal sleep must include a greater number of studied patients with longer follow-up period, in addition to other physiologic and biochemical measurements to answer the questions left open by this study.

\section{AKNOWLEDGMENTS}

In memorian to Juan C. Ugartechea-Hernández MD., D.Sc. pioneer of developmental neurophysiology in Mexico. Thanks to Maggie Brunner M.A., for her help in the preparation of the English version of the article.

\section{CONFLICT OF INTERESTS}

The authors declared that no conflict of interest exists.

\section{REFERENCES}

1. Mirmiran M, Maas YG, Ariagno RL. Development of fetal and neonatal sleep and circadian rhythms. Sleep Rev Med 2003; 7: 321-334

2. Gertner S, Greenbaum CW, Sadeh A, et al. Sleep-wake patterns in preterm infants and 6 months home environment: implications for early cognitive development. Early Hum Dev 2002; 68: 93-102

3. di Pietro JA, Costigan KA, Pressman EK. Fetal state concordance predicts infant state regulation. Early Hum Dev 2002; 68: 1-13

4. Dan B, Boyd S. A neurophysiological perspective on sleep and its maturation. Dev Med Child Neurol 2006; 48: 773-779

5. Shor-Pinsker V, Ugartechea JC, Morales-Caballero F, et al. Characterization of fetal functional states (in Spanish). Ginecol Obstet Mex 1985; 53: 175-180

6. Peirano P, Algarín C, Uauy C. Sleep-wake states and their regulatory mechanisms throughout early human development. J Pediatr 2003; 143: s70-s79

7. Groome LJ, Bentz LS, Singh KP. Behavioral state organization in normal human term fetuses: the relationship between periods of undefined state and other characteristics of state control. Sleep 1995; 18: 77-81

8. di Pietro JA, Hodgson DM, Costigan KA, et al. Fetal neurobehavioral development. Child Dev 1996; 67: 2553-2567

9. Goncalves H, Bernardes J, Rocha AP, et al. Linear and nonlinear analyses of heart rate patterns associated with fetal behavior states in the antepartum period. Early Hum Dev 2007; 83: 585-591

10. Scher MS. Ontogeny of EEG-sleep from neonatal through infancy periods. Sleep Med; in press

11. Capurro H, Konichezky S, Fonseca D, et al. A simplified method for diagnosis of gestational age in the newborn infant. J Pediatr 1978; 93: 120-122

12. Eisenberg de Smoler P, Karchmer S. Four leads in fetal electrocardiography. Am J Obstet Gynecol 1972; 112: 239-245

13. Nijhuis JG, Prechtl HFR, Martin CB, et al. Are there behavioral states in the human fetus? Early Hum Dev 1982; 6: 177-195

14. Freitag W, Schlüter B, Trowitzsch E. Sleep studies in infants-technique and interpretation of polysomnographies (in German). Kinderkrankenschwester 1992; 11: 465-468

15. Villa MP, Calcagnini G, Pagani J, et al. Effects of sleep stage and age on short-term heart rate variability during sleep in healthy infants and children. Chest. 2000; 117: 460-466

16. Prechtl HFR. The behavioral states of the newborn (a review). Brain Res 1974; 76: 185-212

17. Crowell DH, Kulp TD, Kapunaini LE, et al. Infant polysomnography: reliability and validity of infant arousal assessment. J Clin Neurophysiol. 2002; 19: 469-483

18. Hair JE, Anderson RE, Tatham RL, et al. Multivariate data analysis (in Spanish). Madrid: Prentice Hall. 1999

19. Junge HD. Behavioral states and state related heart rate and 
motor activity patterns in the newborn infant and the fetus antepartum. A comparative study. I. Technique, illustration of recordings, and general results. J Perinat Med 1979; 7: 85-107

20. Pillai M, James D. Are the behavioral states of the newborn comparable to those of the fetus?. Early Hum Dev 1990; 22: 39-49

21. Groome LJ, Swibert MJ, Atterbury JL, et al. Similarities and differences in behavioral state organization during sleep periods in the perinatal infant before and after birth. Child Dev 1997; 68: $1-11$

22. Thoman EB, Whitney MP. Behavioral states in infants: individual differences and individual analyses. In: Colombo J, Fagen J, eds. Individual differences in infancy: Reliability, stability, prediction. Hillsdale: Erlbaum. 1990: 113-135

23. Scher MS, Waisanen H, Loparo K, et al. Prediction of neonatal state and maturational change using dimensional analysis. J Clin Neurophysiol 2005; 22: 159-165

24. Kostovic I, Jovanov-Milosevic N. The development of cerebral connections during the first 20-45 weeks' gestation. Semin Fetal Neonatal Med 2006; 11: 415-422

25. Moore JK, Guan YL. Cytoarchitectural and axonal maturation in human auditory cortex. J Assoc Res Otolaryngol 2001; 2: 297-311

26. Kinney HC. Human myelination and perinatal white matter disorders. J Neurol Sci 2005; 228: 190-192

27. Chugani HT, Phelps ME. Maturational changes in cerebral function in infants determined by 18FDG positron emission tomography. Science 1986; 321: 840-843

28. Newberg $A B$, Alavi A. Normal patterns and variants in single-photon emission computed tomography and positron emission tomography brain imaging. Semin Nucl Med 2003; 33 : 42-55

29. Salzarulo P, Fagioli I. Sleep for development or development for waking?- some speculations from human perspective. Behav Brain Res 1995; 69: 23-27

30. Wood SL, Beyer BK, Capon GD. Species comparison of postnatal CNS development: functional measures. Birth Defects Res B Dev Reprod Toxicol 2003; 68: 391-407 